\title{
Enseñanza de las matemáticas en economía: hacia una delimitación de la competencia de matemática de modelación analítica
}

\author{
Teaching Mathematics in Economics: \\ Towards a Delimitation on the Competence \\ of Mathematical Modelling
}

Gustavo Junca Rodríguez*

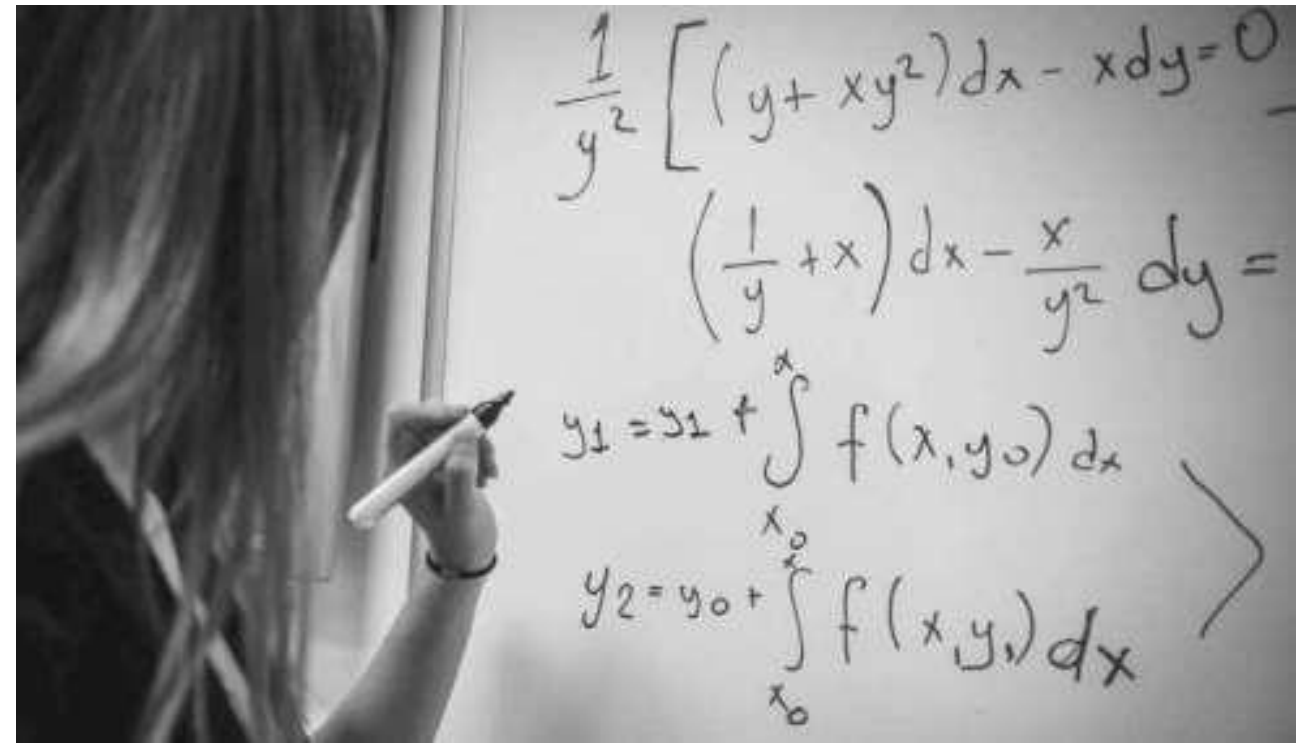

\section{Resumen}

Este documento presenta el desarrollo de la competencia matemática de modelación analítica como una propuesta de enseñanza de las matemáticas. Para ello se define la competencia matemática de modelación analítica dentro de la perspectiva de Acosta y Vasco (2013) y Rychen y Salganik (2003), que caracterizan la competencia a partir de cuatro pilares: actitud, aptitud, sensitividad y reflexividad. Estos

Citar este artículo como: Junca Rodríguez, G. (2019). Enseñanza de las matemáticas en economía: hacia una delimitación de la competencia de matemática de modelación analítica. Revista Papeles, 11(21), 32-54.

Fecha de recibido: febrero 10 de 2019 • Fecha de aceptación: abril 10 de 2019

* Profesor Asociado de la Universidad Nacional de Colombia, y miembro del Grupo de Investigación Interdisciplinaria en Pedagogía del Lenguaje y las Matemáticas (GIIPLYM). Estudiante del Doctorado Interinstitucional en Educación (DIE) de la Universidad Francisco José de Caldas. Agradezco los comentarios del Dr. Carlos Vasco. Cualquier error es responsabilidad del autor. Correo electrónico: gajuncar@unal.edu.co 
elementos son integrados al proceso de modelación matemática. Se presentan los elementos de la competencia matemática de modelación analítica (CMMA) articulados en el Sustrato, la Estructura y la Dinámica. Finalmente se hace una discusión sobre el proceso de representación de los modelos mentales en economía y la enseñanza de las matemáticas a partir del modelo de la CMMA, con especial énfasis en el concepto de marginalidad asociado al objeto de la derivada.

Palabras clave: educación superior, educación matemática, competencias, modelos, teorías.

\begin{abstract}
This paper presents the development of the mathematical analytical modelling competence as a purpose in mathematics teaching. The mathematical analytical modelling competence is defined from Acosta and Vasco's (2013) and Rychen and Salganik's (2003) perspectives, who characterize this competence from four pillars: Attitude, Aptitude, Sensitivity, and Reflexivity. These elements are integrated in the mathematical modelling process. Additionally, elements of the mathematical analytical modelling competence (MAMC), related to Substrate, Structure, and Dynamics, are also presented. Finally, a discussion on the process of representation of mental approaches in economics and mathemathics teaching from this MAMC model is established emphasizing the concept of marginality related to the object of the derivative.
\end{abstract}

Keywords: higher education, mathematical education, competence, models, theories.

\title{
Introducción
}

La discusión y el análisis de las competencias en Colombia se enmarcan desde hace 20 años en la propuesta de evaluar la calidad de la educación a través de indicadores que fueran comparables nacional e internacionalmente, y ya para el año 2000 se cambiaron las pruebas ICFES $^{1}$. Sin embargo, únicamente hasta el 2003 se crean los exámenes para evaluar la calidad de la educación superior a través de los Exámenes de Competencias Académicas de la Educación Superior (ECAES), hoy pruebas SaberPro. De manera paralela, el ICFES inicia con las

1 Instituto Colombiano para el Fomento de la Educación Superior. Ver los trabajos de Bustamante et al. (2002), Bustamante (2003) y Bustamante et al. (2004) para una mirada crítica del concepto de competencia y su contexto en el proceso educativo colombiano. universidades los procesos de acreditación de los programas académicos. Es en este contexto en el cual se inician en las facultades y carreras de economía las primeras reflexiones sobre los aspectos pedagógicos (Fernández, 2005), así como una reflexión sobre la enseñanza por competencias en los distintos programas de economía (Mora, Ramírez y Acuña, 2012).

Desde la perspectiva internacional, esta investigación va en la misma dirección que el proyecto AHELO (Assessment of Higher Education Learning Outcomes), que busca valorar las destrezas genéricas y específicas en ingeniería y economía (OECD, 2012) ${ }^{2}$ y

2 El estudio de destrezas específicas para economía valora los aspectos de la economía aplicada que tiene un claro impacto en las decisiones políticas. 
el examen nacional de desempeño de estudiantes (ENADE) para distintos programas de ciencias sociales y para los programas de ciencias económicas: administración, contaduría y economía, que llevará a cabo en 2012 el gobierno de Brasil (Ministério-Educação \& INEP, 2012).

En los trabajos de Fernández (2005), Bernat, Jácome, Mejía, Torres y Torres (2010) y Mora, Ramírez y Acuña (2012) de manera explícita se hace referencia al conocimiento y uso de objetos matemáticos para abordar los problemas de la realidad económica. Esto supone una matematización que integre conceptos económicos con objetos matemáticos para la construcción de modelos y teorías en economía. Allí la representación aparece primero para pasar de subprocesos de la realidad económica a modelos mentales. Luego de la modelación se pasa a la teorización, que de acuerdo con Vasco (2014) corresponde a la representación del modelo mental en un registro articulado, es decir, verbal-oral, verbal-escrito o simbólico de las matemáticas (aritmética, álgebra o cálculo).

Sin embargo, el problema de la didáctica es que los modelos mentales son difíciles de comunicar, de allí la necesidad de una serie de estudios sobre la didáctica de las matemáticas o la didáctica de la economía para entender el desarrollo de las competencias en general y la competencia matemática de modelación analítica, en particular. De lo contrario, únicamente bastaría con el estudio de las matemáticas, la economía o la lingüística. Es posible que los estudiantes se aprendan las teorías, pero no sabemos en qué modelos las interpretan; más aún, no tenemos certeza de si las interpretan o solo las repiten.

Tenemos así que para Ministerio-Educación (1998) y Pisa (2009), el aprendizaje de las matemáticas debe permitir la aplicación de esos

Los países seleccionados para el estudio son Bélgica, Egipto, Italia, México, Holanda, Rusia y Eslovaquia. conocimientos en contextos distintos a los del ámbito escolar. De igual manera, los conocimientos en matemáticas aprendidos durante el proceso de formación del economista deben trascender el ambiente universitario, deben permitirle la toma de decisiones, enfrentarse y adaptarse a nuevas situaciones e interactuar de manera efectiva con otros. Debemos entender cómo esas teorías y modelos los relacionan con la realidad económica. Así, una visión global e integral está compuesta de tres grandes aspectos, como lo expresa el documento de lineamientos curriculares en matemáticas del Ministerio de Educación Nacional (1998):

- Procesos generales del aprendizaje: razonamiento; resolución y planteamiento de problemas; comunicación; elaboración, comparación y ejercitación de procedimientos; y modelación.

- Conocimientos básicos relacionados con procesos específicos que desarrollan el pensamiento matemático y con sistemas propios de las matemáticas, como son: pensamiento numérico y sistemas numéricos; pensamiento espacial y sistemas geométricos; pensamiento métrico y sistemas de medida; pensamiento aleatorio y sistemas de datos; y pensamiento variacional y sistemas algebraicos y analíticos.

- Contexto de situaciones problemáticas de las matemáticas mismas, de la vida diaria y de otras ciencias.

Consideramos así que los procesos de modelación, el pensamiento variacional y los sistemas algebraicos y analíticos permiten la resolución de situaciones problemáticas en economía y de alguna manera son transversales a los otros procesos generales y de conocimientos básicos. Concentrarnos en ellos nos permitirá responder a nuestra pregunta de investigación, es decir, cómo se puede evidenciar el desarrollo de la competencia matemática de modelación analítica en economía, en el proceso de aprendizaje de los conceptos de la marginalidad y de la derivada. 


\section{Enseñanza de las matemáticas en economía}

Para la disciplina económica no ha existido una reflexión sistemática sobre los procesos de enseñanza y aprendizaje, pero durante los últimos 12 años han emergido varios proyectos con el fin de proporcionar recursos docentes para mejorar la enseñanza de la economía. Los recursos varían desde estrategias y materiales para ser utilizados por docentes, profesores asistentes, y estudiantes, así como reflexiones sobre la actividad docente y múltiples casos de estudio sobre diversos tópicos. Dentro de este contexto han surgido el International Review of Economics Education (IREE) en 2003 y el Computers in Higher Education Economics Review (CHEER) en 1986, que dan cuenta de la creciente importancia de la reflexión sobre los procesos de enseñanza y aprendizaje en economía.

Esta reflexión sobre la práctica docente ha estado precedida por numerosos estudios que evidencian las deficiencias en la enseñanza de la economía a nivel del pregrado ${ }^{3}$, según los cuales muchos estudiantes graduados no entienden algunos conceptos básicos en economía y consideran en general que han recibido una educación de baja calidad (Hartman, 1978; Siegfried y Fels, 1979; Colander y Klamer, 1987; Allgood y Walstad, 1999a, 1999b, 2005; Becker, 1997; Becker y Watts, 1999; Walstad, 2001; Salemi, Siegfried, Sosin, Walstad y Watts, 2001). Algunas de las críticas resaltan los problemas sobre el énfasis en los aspectos teóricos, así como una muy baja conexión con los problemas del mundo real. Es decir, los modelos ${ }^{4}$ utilizados son poco

3 Ver Hansen (1991) para una revisión sobre la educación y entrenamiento de los estudiantes de doctorado de los programas de economía en Estados Unidos. Siegfried y Stock (2007) para un análisis sobre la profesión de los estudiantes de los programas de doctorado en economía.

4 El concepto de modelo utilizado en la disciplina económica parece incluir el concepto de teoría realistas, y cuando buscan serlo se tornan excesivamente complejos (Heyne, 1995; Siegfried, Bartlett, Hansen, Kelley, McCloskey and Tietenberg, 1991) $)^{5}$.

Sin embargo, a pesar de la multitud y variedad de trabajos ya publicados, se puede afirmar que aún no ha existido una reflexión sistemática sobre los aspectos de la didáctica de las matemáticas en economía. La reflexión sobre didáctica de la matemática en economía es apenas incipiente y se centra en un conjunto de ayudas, computacionales o no, que permiten al estudiante entender los conceptos matemáticos y su aplicación a los problemas económicos (Hey, 2005; Raymond, Raymond y McCrickard, 2008; Vazquez-Cognet, 2008; Gorry y Gilbert, 2015; Pezzino, 2016).

Otro conjunto de trabajos buscan analizar la relación entre el desempeño en los cursos de matemáticas y el desempeño en los cursos introductorios de economía. En efecto, los trabajos de Ballard y Johnson $(2004,2005)$ analizan dicha relación e incorporan los aspectos de género para explicar diferencias en el desempeño. Didia y Hasnat (1998) restringen su análisis a los cursos introductorios de finanzas. Kherfi (2008) presenta sus resultados para Oriente Medio. Butler, Finegan y Siegfried (1998) muestran que el desempeño en los cursos de microeconomía es mejor cuando los estudiantes han tomado más cursos de cálculo y su relación es casi nula con respecto a los curso de macroeconomía. Gery (1970) explica la paradógica relación negativa entre el desempeño en matemáticas y el rendimiento

utilizado por los teóricos de las ciencias desde la perspectiva estructuralista. Ver Balzer y Moulines (1996), Balzer, Moulines y Sneed (1986, 2000), Diez y Moulines (1997).

5 Ver Lora \&amp; Ñopo (2009) y Colander \&amp; Nopo (2011) para un análisis de la estructura curricular y la formación de los economistas América Latina. 
en los cursos introductorios en economía. Benedict y Hoag (2002) muestran cómo tener mejores habilidades en matemáticas reduce la ansiedad y mejora el desempeño en los cursos de introducción a la economía. Zweig y Dawes (2000) proponen una metodología que combine la aproximación cualitativa y cuantitativa en la enseñanza de los cursos introductorios de economía 6 . Lagerlöf y Seltzer (2009) miran el efecto de los cursos remediales de matemáticas sobre el aprendizaje de la economía. Stavely-O'Carroll (2015) muestra el efecto positivo de las demostraciones en clase y las discusiones virtuales en el desempeño de los estudiantes para el curso de principios de economía. Dubas y Toledo (2016) muestran cómo el uso de la Taxonomía de Marzano para discriminar un desarrollo progresivo del pensamiento en principios de economía tiene un impacto positivo en el desarrollo de un nivel alto de pensamiento.

Algunos trabajos proponen elementos pedagógicos para introducir el concepto de marginalidad en distintos contextos económicos, como es el caso de Asano (2006), Rigall-I-Torrent (2011), Beattie y LaFrance (2006) y Raboy (2016).

Finalmente, la mayor parte de la literatura se ha centrado en los aspectos didácticos, es decir, qué enseñar y cuál es la relevancia de las matemáticas en economía y su pertinencia para analizar los problemas de la economía real. El trabajo de Chen (2008) plantea la necesidad de utilizar modelos mas complejos para abordar los problemas económicos, y Backhouse

6 Otros trabajos analizan el desempeño general de los estudiantes en el programa con relación a su desempeño en el pasado y su desempeño con relación a la cohorte a la cual pertenecen como es el caso de Naylor \&amp; Smith (2004), mientras que Nolan \&amp; Ahmadi-Esfahani (2007) analizan el desempeño de los estudiantes de economía agrícola con relación a los programas de economía y comercio. Guest \&amp; Duhs (2002) desempeño en los programas de economía de las Universidades de Australia.
(1998) enfatiza en los aspectos no formales entre matemáticas y economía. Otros trabajos plantean una discusión sobre la importancia y pertinencia de las matemáticas en economía: Bennett \& Johnson (1979); Grubel \& Boland (1986); Kantorovich (1989); Katzner (2003); Phillips (2007); Rosser (2003); Samuelson (1994). Mientras que Sproule (2015) propone la utilización de la función cuadrática para la enseñanza de los conceptos básicos en microeconomía en cambio de la tradicional función Cobb-Douglas.

Desde la educación matemática y la didáctica de las matemáticas hay tres trabajos relevantes (Fonty Ramos, 2005, 2008; Ramos, 2006) donde se aborda el proceso de contextualización de las funciones en los procesos de enseñanza y aprendizaje de las matemáticas en las ciencias económicas (administración, contaduría, economía) ${ }^{7}$. Ellos muestran cómo dicha contextualización es relevante para el aprendizaje de las matemáticas en un ambiente dominado por un enfoque formalista y estructuralista.

Desde el enfoque ontosemiótico (EOS) de la cognición y la instrucción matemática se plantean los criterios de idoneidad y valoración de los cambios del profesor de introducción a las matemáticas al enseñar el objeto función dentro del contexto de las ciencias económicas y el papel que juegan los objetos personales matemáticos y didácticos en dicho proceso de cambio personal e institucional. Su trabajo se centra en el papel de los profesores de matemáticas para formar profesionales competentes en ciencias económicas, de allí la importancia de la enseñanza de los objetos matemáticos, en este caso de las funciones, dentro de un contexto económico. Es así como el trabajo de Ramos (2006) aborda no solo las competencias de los profesores para resolver problemas contextualizados de funciones, sino también las

7 La investigación de Ramos (2006) se llevó a cabo en la Facultad de Ciencias Económicas y Sociales de la Universidad de Carabobo en Venezuela. 
competencias de los estudiantes para resolver este tipo de problemas.

Los trabajos de Font \& Ramos (2005, 2008) complementan los trabajos sobre el análisis de distintos aspectos didácticos y matemáticos en los procesos de enseñanza y aprendizaje de la derivada en la formación de profesores de matemáticas desde el enfoque ontosemiótico (EOS) de la cognición y la instrucción matemática (Badillo, Azcárate y Font, 2011;
Font, 2000, 2005, 2009, 2010; Font, Robles, y Del-Castillo, 2011a; Godino, Font, Pino-Fan, y Castro, 2011).

Por los antecedentes anteriormente mencionados, se justifica un estudio que integre la competencia matemática de modelación analítica en economía y, más aún, su relevancia cobra importancia para el caso colombiano, donde este tipo de análisis aún están por desarrollarse.

\section{Modelo para el desarrollo de la CMMA}

Podríamos describir el desarrollo de la competencia matemática de modelación analítica (CMMA) como un proceso que incluye tanto las fases de modelación como la articulación con los elementos que caracterizan la competencia desde el enfoque de las disposiciones. Siguiendo la metodología de Vasco (2014) ${ }^{9}$, haremos una descripción del Modelo CMMA, es decir, de sus elementos, de su estructura y de su dinámica. En la siguiente sección nos centraremos fundamentalmente en los elementos teóricos de dicho modelo. En el trabajo de Vasco (2014) se hace una distinción rigurosa entre modelos y teorías. Nosotros presentamos acá el modelo mostrando cómo el Sustrato corresponde a los elementos relevantes del modelo potencial. Es decir, la lista de elementos que conforman el modelo. Una segunda formulación corresponde a la estructura en la cual se relacionan las partes del modelo. Y finalmente está la representación

8 Para ver una versión más completa del Desarrollo del modelo de Competencia Matemática de Modelación Analítica en Economía ver el trabajo doctoral de Junca (2018).

9 Ver Vasco (2014) y Junca (2018) para una presentación rigurosa de la metodología de modelos y teorías. En particular, Junca (2018) presenta un análisis comparativo de los enfoques de modelos y teorías a partir de la teoría general de procesos y sistemas, y desde la teoría estructuralista de la ciencia. del modelo dinámico, donde se muestra cómo cambia el modelo en sus estructuras o sus partes. Como veremos en nuestro caso queremos mostrar como el modelo de la CMMA en economía se desarrolla en una posible ruta.

\section{Sustrato o conjunto de elementos del modelo CMMA}

Desde la Teoría General de Procesos y Sistemas (TGPS) el sustrato corresponde al conjunto de elementos o componentes que forman parte del modelo seleccionados de un trasfondo difuso $^{10}$. Debido a que estamos planteando un modelo de CMMA para un individuo (estudiante de economía), los principales elementos que forman el sustrato del modelo podemos agruparlos en algunas categorías.

\section{Trasfondo}

- Primer nivel: del individuo y su subjetivida $^{11}$. Los procesos mentales y psicológicos del individuo se viven como provocados por procesos "allá afuera", es decir, por problemas percibidos como realidad individual

10 El sustrato correspondería al modelo potencial parcial dentro de la Teoría Estructuralista de la Ciencia (TEC).

11 Correspondería a la faceta que en el Enfoque Onto Semiótico (EOS) se llama "cognitiva". 
o subjetiva desde el punto de vista del observador distante ("bystander") que los "sufre" o "padece" y trata de reconstruirlos "aquí adentro". Aquí podemos entender "problemas" como procesos percibidos como extraños, conflictivos, contradictorios, dolorosos, repugnantes, discordantes, desagradables, perturbadores para el sujeto.

- Conjunto de procesos y problemas que conforman lo real: procesos educativos, procesos políticos, procesos sociales, etc.

- Subconjunto de procesos que conforman lo real económico.

- Segundo nivel: realidad compartida por un grupo de interés: percepción, introyección y proyección de los procesos y problemas "allá afuera". Es decir, los modelos y teorías mentales de cómo los individuos introyectan o internalizan y, luego, externalizan o proyectan como procesos "allá afuera" a través de sus modelos y sus teorías, que comparten con otros individuos de su grupo para definir lo que llaman la realidad y la realidad económica, desde el punto de vista del grupo de interés que comparte ofertas, demandas, costos y beneficios.

- Realidad económica compartida por los individuos del grupo.

- Tercer nivel: problemas relevantes de la realidad económica a nivel local, nacional e internacional. Este es el nivel de la comunidad de expertos ${ }^{12}$.

- Realidad económica compartida por la comunidad de economistas (incluyendo

12 Correspondería a la faceta epistémica en el Enfoque Onto-Semiótico (EOS). Alude a los aspectos del Trasfondo del Tercer Nivel que están institucionalizados en una comunidad académica de expertos. Puede compararse con el paradigma de Thomas Kuhn y con el Núcleo Central en Lakatos y en la TEC. Estos insumos para el Tercer Nivel suelen llamarse "recursos del medio", que incluyen los insumos ya producidos y las personas que los saben utilizar ("recursos materiales" y "recursos humanos"). los estudiantes de distintos niveles) que pretenden llegar a "lo real", a la realidad objetiva.

- Realidad económica compartida por cada individuo que conforma la comunidad de economistas.

- Conjunto de problemas relevantes de la realidad económica, considerada como objetiva o "real" por la comunidad de economistas.

\section{Actores o agente(s) noético-} semióticos del transfondo del tercer nivel

- Expertos en economía, profesores, divulgadores, funcionarios y asesores.

- Estudiantes de economía que sean capaces de identificarse y adoptar los puntos de vista de los distintos actores o agentes económicos, con sus:

- Competencias. Entre ellas la competencia matemática de modelación analítica.

- Modelos y teorías.

- Noesis, que les permiten generar modelos, formular teorías y razonar con sus modelos y teorías; y semiosis, que les permiten representar modelos e interpretar teorías a través de distintos registros semióticos (Duval, 1995/2004).

\section{Otros insumos del trasfondo del tercer nivel}

- Material bibliográfico y audiovisual.

- Software especializado para cálculos y simulaciones.

- Conjunto de asignaturas ya cursadas y conjunto de asignaturas disponibles, con sus respectivos docentes y programas, estudiantes y egresados que ya los cursaron. Posgrados y grupos de investigación que desarrollan y profundizan. 
- Información cuantitativa y cualitativa sobre el problema(s) de la realidad económica a tratar.

- Otras herramientas, materiales, información, etc.

- Otros procesos institucionales de cada facultad de economía (Procesos de inscripción, programación de asignaturas, currículo, procesos administrativos, etc).

\section{Estructura del modelo CMMA}

En la TGPS, la estructura es la red de relaciones, nexos, enlaces o restricciones entre los componentes o elementos del sustrato del sistema.

Al plantear la estructura de nuestro modelo de CMMA queremos enfatizar las posibles relaciones que existen entre los elementos planteados en el sustrato o conjunto de componentes del modelo. El Gráfico 1 muestra una representación estilizada estática y sincrónica (no-dinámica) del modelo de CMMA.

Como se muestra en el Gráfico 1, con relación al trasfondo se busca representar las relaciones de introyección y proyección entre los procesos de la realidad "allá afuera", incluyendo los procesos y problemas económicos de producción, distribución, consumo, etc., desde y hacia la realidad económica entendida como los modelos y teorías compartidos por la comunidad de economistas o grupos de ellos, así como los modelos y teorías de cada miembro de la comunidad, incluidos los estudiantes de economía o futuros economistas como aspirantes a participar plenamente en ellas.

De esa realidad económica compartida resulta una primera interpretación, a partir de los modelos y teorías mentales y de la proyección delos procesos económicos, que denominamos conjunto de procesos y problemas relevantes acerca de la realidad económica. Esa realidad económica, si bien forma parte del trasfondo,

Gráfico 1. Representación estilizada no-dinámica del modelo de CMMA

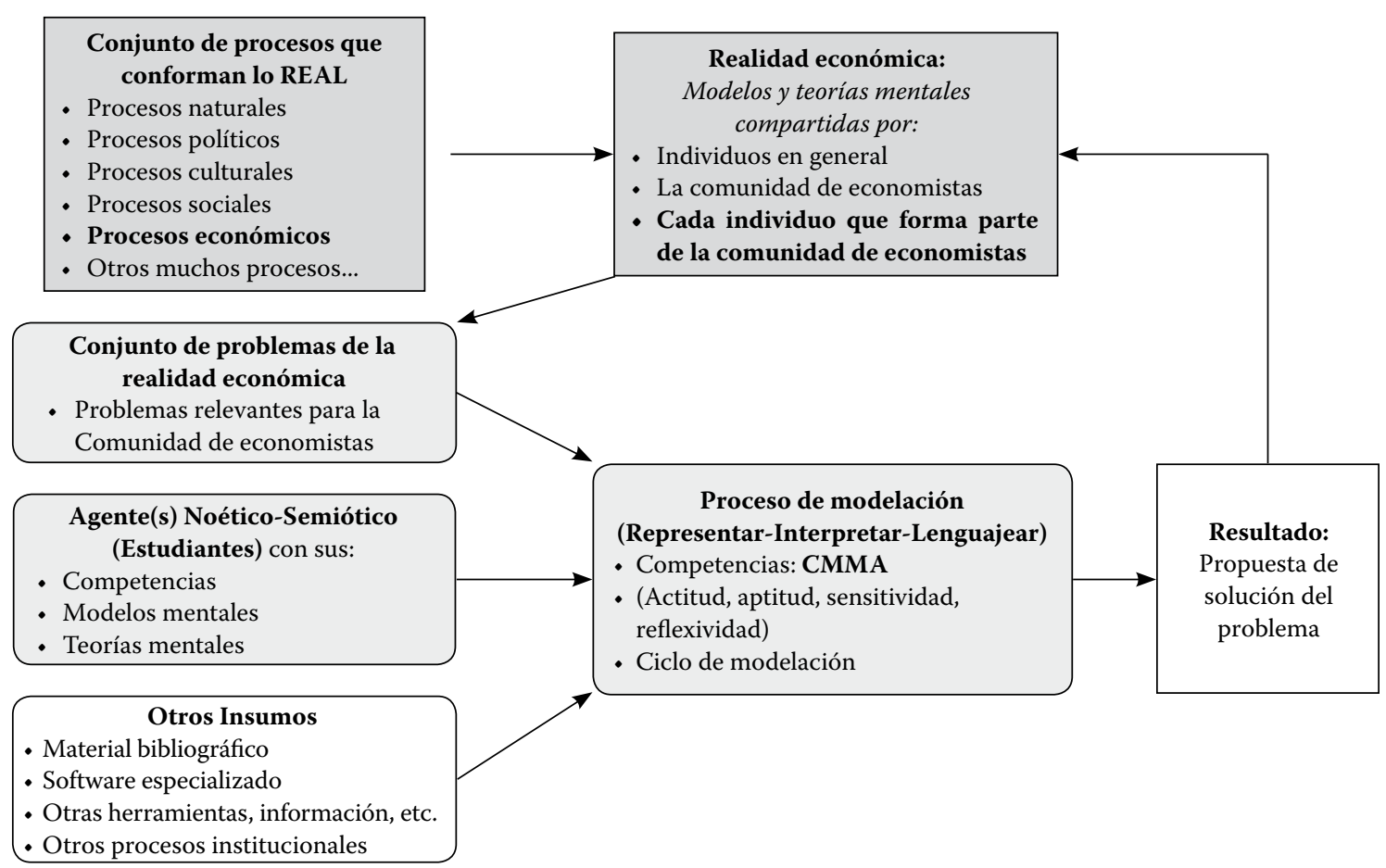

Fuente: elaboración propia. 
claramente es un insumo para el proceso de modelación.

Los otros insumos los hemos agrupado en otros dos conjuntos. En primer lugar está el estudiante de economía, que hemos caracterizado como un agente noético-semiótico, en proceso de incorporación a la comunidad de economistas del tercer nivel del trasfondo, siguiendo a Vasco $(2011,2014)$. Además de suponer que nuestro estudiante tiene la capacidad de representar, interpretar y "lenguajear", este individuo tiene unas competencias, así como sus propios modelos y sus propias teorías, que va construyendo y desarrollando primero durante su formación de economista y luego en su vida profesional.

En tercer lugar, suponemos que el individuo tiene a su alcance otro conjunto de herramientas y recursos, como material bibliográfico, software especializado, que incluye, si fuera necesario distinguir, libros y material digital. De igual manera puede tener al alcance información cuantitativa y cualitativa acerca del problema a tratar. Finalmente, suponemos que existe un apoyo institucional que incluye un conjunto de procesos académicos y de personas (recursos humanos).

Estos elementos del ambiente interactúan en mayor o menor medida en el proceso de

\section{Además de suponer que nuestro} estudiante tiene la capacidad de representar, interpretar y "lenguajear", este individuo tiene unas competencias, así como sus propios modelos y sus propias teorías, que va construyendo y desarrollando primero durante su formación de economista y luego en su vida profesional. modelación, por lo que hemos destacado en el gráfico, un conjunto de acciones como representar, interpretar y "lenguajear". De igual manera hay una interacción entre las competencias, en particular la CMMA y el ciclo de modelación.

Finalmente, se espera que se tenga un resultado que permita interpretar y plantear una o varias soluciones al problema económico que se ha abordado y que dicha solución se pueda contrastar o implementar para afectar (atenuar, acelerar, cambiar, etc.) los procesos económicos y la proyección que comparte la comunidad de economistas, es decir, la realidad económica. Una vez realizada esta última etapa, tendremos que determinar si hay que refinar, modificar o cambiar el proceso de modelación a partir de su impacto en el problema que se buscaba resolver.

Siguiendo a Vasco $(1995,2014)$ en un ejercicio cronotópico, buscaremos representar las relaciones al interior del proceso de modelación, desagregando los elementos espaciales y temporales que intervienen y buscando evidenciar las posibles relaciones entre dichos elementos.

Como se muestra en el Gráfico 2, se presentan las relaciones al interior del proceso de modelación entre la CMMA y el Ciclo de Modelación. Mientras en esta primera aproximación tenemos que la actitud o inclinación está relacionada con todas las fases del ciclo de modelación, la aptitud está presente principalmente en la fase de matematización y en el trabajar matemáticamente.

Por su parte, consideramos que la sensitividad está presente principalmente en las fases de entender el problema económico y plantearlo en términos de la teoría económica y las matemáticas adecuadas.

Por último, la reflexividad está principalmente en la fase de interpretación de las matemáticas en la teoría económica así como en la validación del modelo para ver si la propuesta de solución responde al problema planteado. 
Gráfico 2. Representación no-dinámica del proceso de modelación

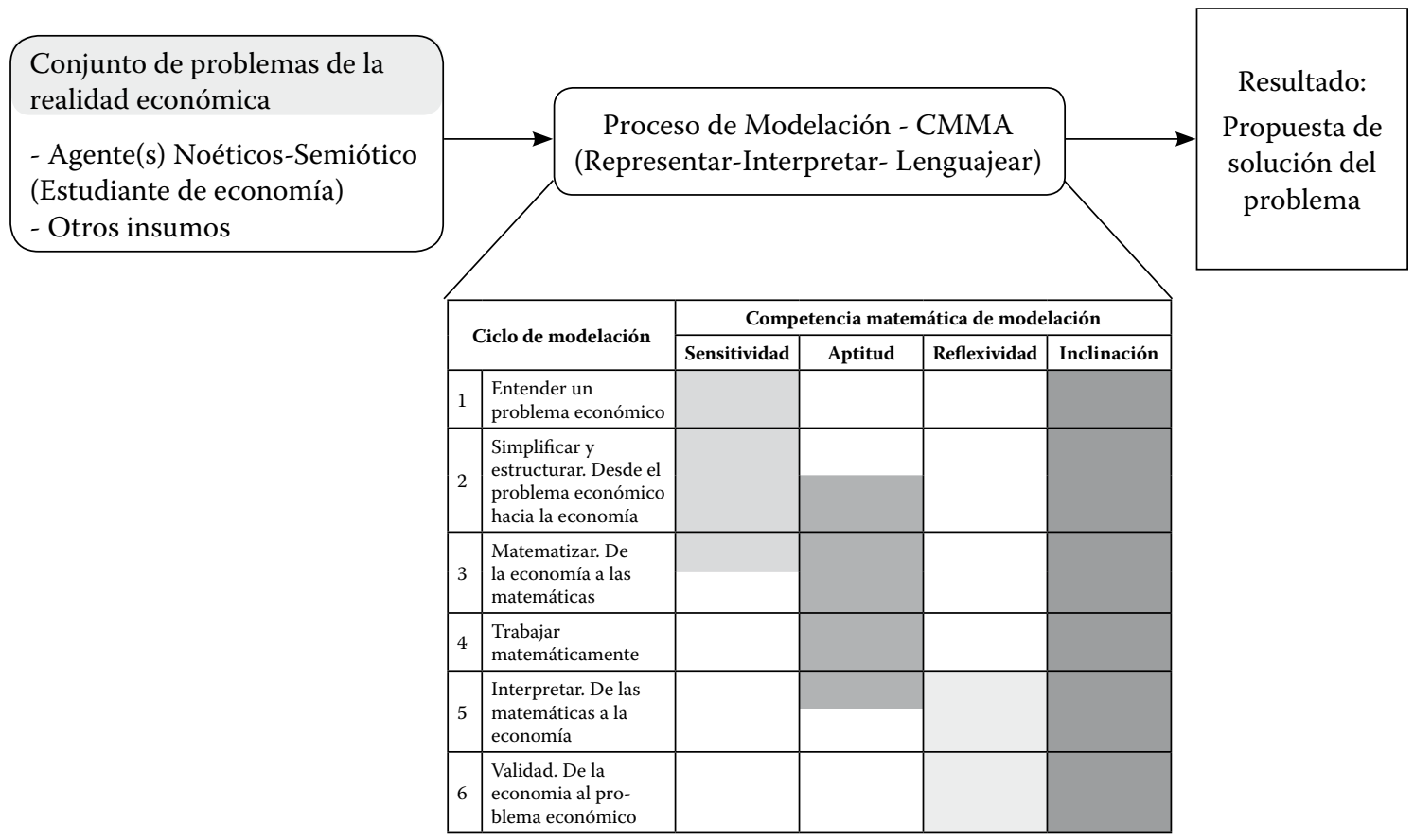

Fuente: Junca (2018)

\section{Dinámica del modelo CMMA}

Cuando hablamos de dinámica del modelo de CMMA debemos tener en cuenta los cambios en los distintos elementos que forman parte del sustrato. Es decir, cambios en el trasfondo, en los insumos y sus relaciones que hemos denominado la estructura, y en el resultado debido a los procesos de retroalimentación que resultan de la solución del modelo y su contrastación. Así, la dinámica podría capturarse a través de tres factores distintos.

- Cambio en los problemas de la realidad económica: es decir, un cambio no solo en los procesos económicos en sí mismos, sino en la manera como esos cambios son proyectados en la realidad económica; es decir, los cambios en los modelos y teorías compartidos por la comunidad de economistas. Algunos ejemplos de cambios sustanciales podrían ser: la revolución industrial, la revolución de las telecomunicaciones, la crisis económica de los años
30, las hiperinflaciones en América Latina en los años 80, el proceso de globalización y apertura de las economías, entre otros muchos.

- El mismo desarrollo progresivo, en el caso de los estudiantes de la CMMA, como se muestra en el Gráfico 3, se presenta una manera entre muchas otras, de cómo va emergiendo los componentes de la CMMA en su desarrollo en el caso de los estudiantes de economía. En el ejemplo se supone que van emergiendo dichos componentes en distintos grados y a su vez pueden permitir un proceso más refinado del ciclo de modelación.

- Refinamiento en los modelos y teorías. Una tercera fuente de cambio, presentada en el Gráfico 3, es el refinamiento de los Modelos y Teorías que pretenden representar, interpretar y "lenguajear" el problema de la economía real que se ha planteado. Una vez se lleva a cabo la validación y se analizan 
Gráfico 3. Representación dinámica del proceso de modelación

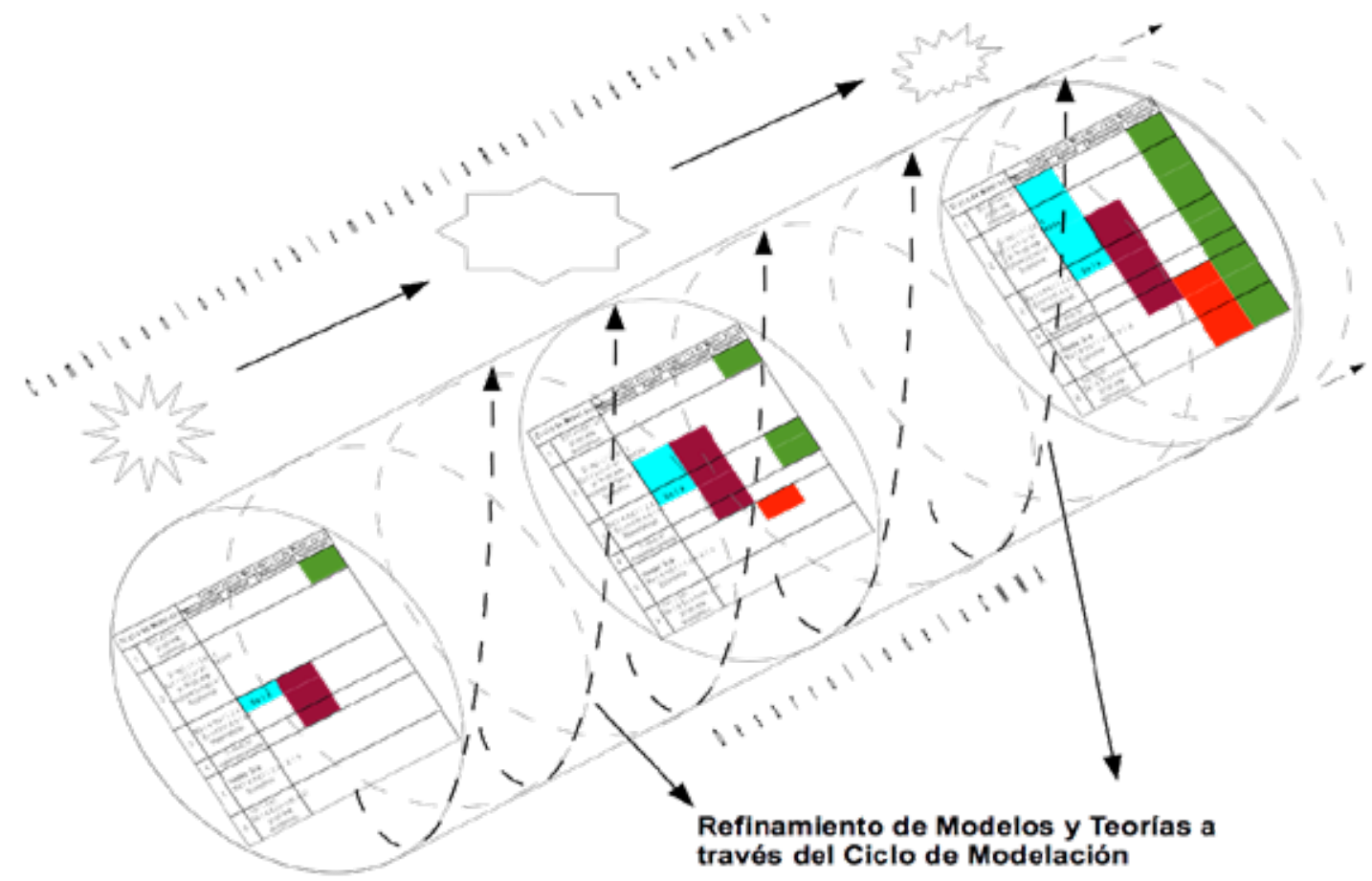

Fuente: Junca (2018)

críticamente los alcances y limitaciones de los modelos y teorías, la posibilidad de refinar dichos modelos y teorías permitirá tener una nueva propuesta de solución al problema.
Claramente, es muy probable que al tiempo se perciban cambios institucionales y cambios en otros procesos no económicos que afecten directamente tanto la realidad económica como el problema a resolver.

\section{Representar el modelo mental por medio de una teoría económica}

Los dos elementos articuladores entre los modelos y las teorías son los morfismos de representación e interpretación (Vasco, 2014). En este apartado abordaremos el morfismo de representación que relaciona el modelo CMMA con la teoría.

De acuerdo con Mockus (1988), el concepto de representar, antes de Descartes, se remonta a la interpretación en el arte de la representación como acontecimiento vinculante que no puede separar la acción de representar de aquello que se representa, que se pone en escena. Más aún, en el caso de la noción cristiana, el rito de la eucaristía actualiza la muerte y resurrección de Cristo. El representar es como volver a hacer presente, una y otra vez, un acontecimiento a través de la puesta en escena de un rito.

De igual manera, la obra de teatro no se lee, se representa, se actúa. Aquello que se representa cobra vida durante la representación. Igual que la representación como recurso en la retórica, es decir, de poner ante los ojos, como estando 
nuevamente presente, en otra dimensión espacio-temporal, aquello que fue representado y que está de nuevo representándose.

En Descartes, de acuerdo con Mockus (1988), la noción de representar no es solo una acción externa al individuo, sino interna. Es decir, las representaciones internas nos permiten tomar conciencia de los procesos "allá afuera", de lo real. Algo más que una mera percepción sensorial, pues son objeto de nuestro pensamiento. La acción de representar y disponer en la mente nos permite construir imágenes y discursos mentales a través del pensamiento, lo que Vasco (2014) ha llamado modelos y teorías del individuo, que forman su realidad. De acuerdo con Mockus (1988), esta toma de conciencia en Descartes da comienzo a la modernidad. Más aún, en el texto de Mockus (1988) se podría también hablar de representar y disponer de manera externa en el planteamiento de Descartes, es decir, a través de la extensionalización de la realidad en variables que pueden ser medidas, cuantificadas. Esa matematización implica representar y disponer externamente, es decir, sobre una hoja de papel, las variables como magnitudes y las relaciones entre estas variables. Mockus (1988) no distingue entre estos dos tipos de representaciones; más aún, lo que denominamos representación externa es una reestructuración de la representación interna ${ }^{13}$.

Los modelos mentales son las representaciones del individuo de los procesos "allá afuera" con sus relaciones, es decir, dispuestos "allí adentro", como si estuvieran dispuestos en una hoja de papel, con un orden y una jerarquía determinados por el individuo, de manera que conforman su realidad.

La triada propuesta por Vasco (2014) es la de representador-representante-representado

13 Ver Mockus (1988), donde distingue las dos representaciones dejando claro que la uno no es la copia o duplicado exacto de la otra. junto con la acción de representar. Desde su perspectiva de la TGS y TGP, tenemos que:

- Representador es el agente noético-semiótico, en nuestro caso con sus competencias y su realidad (sus modelos y teorías).

- Representante es el sistema como modelo, con sus teorías, es decir, con sus relaciones y operaciones.

- Representado son los procesos o subprocesos, lo real.

Es así como esta tríada está a la base de la TGPS. Podríamos haber utilizado estas categorías en el Sustrato para enumerar los elementos que conforman el sistema como modelo.

Siguiendo a Duval (1995, 2004), una primera distinción que mantiene Vasco (2014) es la de representaciones internas y representaciones externas.

Una primera acción de representar la lleva a cabo el agente noético al llevar a cabo las representaciones internas de los procesos y subprocesos en imágenes como modelos mentales y teorías mentales. Como acto consciente en el sentido de Descartes que nos permite distinguir lo real (allá afuera) de la realidad (acá adentro).

Obsérvese que los modelos y teorías económicas aprendidos durante la carrera por los estudiantes de economía, así como su articulación con las aplicaciones matemáticas y los objetos matemáticos en general, forman parte de este conjunto de representaciones internas que forman la realidad del agente noéticoseimiótico, del estudiante de economía. Los procesos de enseñanza y sus aprendizajes le permiten representar internamente como imágenes los modelos y teorías, para luego ser representadas e interpretadas en su vida profesional, para volverse representaciones externas.

Las representaciones externas corresponden a los modelos y teorías que, como diría Mockus (1988), permiten representar y disponer de 
manera externa sobre un papel, aunque no en el sentido amplio utilizado por Vasco (2011, 2014) o, como diría Duval $(1995,2004)$, una representación externa y materializada como producto de los registros semióticos de representación. Es así como el agente noético-semiótico posee el sistema productor de representaciones semióticas materializadas, con las cuales hace sus representaciones semióticas de los procesos y subprocesos, es decir, materializa los modelos y teorías no necesariamente como una copia, un duplicado, como distingue Mockus (1988), sino como lo que es, una representación externa de una representación interna.

Es así como representar los modelos internos en las teorías internas consiste en explicar las relaciones entre los elementos que conforman la imagen del modelo interno a través del discurso mental, como habla silenciosa o subvocal en lo que un agente-hablante experimenta como discurso interior con sentido, el cual no puede adjudicarse ni al modelo ni a la teoría sino a la interpretación de cada enunciado de la teoría en el modelo (Vasco, 2014, p. 46).

Como mencionamos al comparar la TGPS con la TEC, para Vasco, la teorización sobre las relaciones del modelo, están presentes desde el comienzo, en las representaciones internas. Más aún, a esa teorización contribuyen los modelos económicos y las teorías económicas compartidas por los economistas, que el estudiante ha aprendido durante su formación como economista.

De igual manera, el estudiante al hacer las representaciones semióticas materializadas del modelo busca, a través de la teoría, explicar las relaciones entre los elementos de dicho modelo. Para ello utiliza el lenguaje verbal, los objetos matemáticos ${ }^{13}$, los dibujos, las figuras, la representación del procesamiento de datos a través de software estadístico y econométrico, etc.

\section{Enseñanza de las matemáticas a partir de la CMMA}

El punto de partida de la evolución conceptual que llevó a precisar el concepto de competencia que utilizamos en esta investigación se inició con una descripción amplia de competencia elaborada por Vasco (2000) para la capacitación de los docentes en el desarrollo y evaluación de las competencias de sus estudiantes, con el fin dar respuesta a los cambios en los exámenes del Icfes (ahora llamados Saber Once) a partir del año 2000:

Una competencia es un conjunto de conocimientos, habilidades, actitudes, comprensiones y disposiciones cognitivas, metacognitivas, socioafectivas y psicomotoras, apropiadamente relacionadas entre sí, para facilitar el desempeño flexible, eficaz y con sentido de una actividad o de cierto tipo de tareas en contextos relativamente nuevos y retadores (Vasco, 2004).
A partir de ese texto se inició una serie de discusiones en seminarios doctorales y en proyectos de investigación para tratar de refinar los conceptos de comprensión y disposición con la teoría de la enseñanza para la comprensión - Teaching for Understanding- del Proyecto Cero de Harvard y con la teoría de las disposiciones cognitivas (Perkins et al, 2000; Perkins y Ritchhart, 2004), en particular con la atención, entonces muy novedosa, a la sensibilidad o sensitividad como "radar" de detección de oportunidades.

Los cuatro aspectos conceptuales de la competencia que se fueron decantando desde el estudio de los dos volúmenes de la OCDE

14 Es importante mencionar que a los objetos matemáticos también podemos acceder a través de sus representaciones. 
editados por Rychen y Salganik $(2001,2003)$ y el desarrollo de la tesis de Acosta y del presente estudio, hasta el libro de Acosta y Vasco (2013, son los siguientes:

- La aptitud, que incluye tanto habilidades ("know-how") como conocimientos ("know-that").

- La actitud o inclinación, que incluye los aspectos afectivos, como la motivación, el autoconcepto positivo y otras actitudes apropiadas.

- La sensitividad, que permite detectar las oportunidades de transferir las habilidades y conocimientos a tareas relativamente diferentes en contextos nuevos y retadores.

- La reflexión o reflexividad, que incluye los aspectos metacognitivos y metaafectivos que permiten el monitoreo de la actividad, la ubicación de los tres aspectos anteriores en contextos y sentidos más amplios, la atención a las relaciones fin-medios y costo-beneficio, y la previsión de consecuencias futuras.

La presencia de estos cuatro aspectos permite distinguir la competencia de las meras capacidades ("capacities" y "capabilities"), aptitudes, habilidades, destrezas y talentos ("abilities", "skills", "gifts"), sin pretender que la competencia se extienda todavía a la experticia o al virtuosismo (Acosta y Vasco, 2013, p. 37).

En este artículo utilizaremos "el modelo de la mesa de cuatro patas", llamado así en comparación con "el modelo de la mesa de tres patas" del libro de Acosta y Vasco (2013) y en Vasco (2000), en donde no se había fijado la atención en los aspectos reflexivos señalados por Rychen y Salganik (2003). Es así como, siguiendo a Acosta y Vasco (2013) y Rychen y Salganik (2003), definimos competencia como: el conjunto de conocimientos, habilidades, actitudes, comprensiones y disposiciones cognitivas, metacognitivas, socioafectivas $y$ psicomotoras, apropiadamente relacionadas entre sí, para facilitar el desempeño reflexivo, flexible, eficaz y con sentido de una actividad o de cierto tipo de tareas en contextos relativamente nuevos y retadores. Como veremos más en detalle, este es nuestro enfoque de competencias utilizado en el desarrollo del modelo y la teoría de la CMMA.

Además, en esta investigación estamos interesados en la competencia de modelación matemática del economista desarrollado a través de su formación universitaria. Tenemos así cómo un economista se inserta en el mercado laboral y enfrenta un conjunto de problemas relacionados con los procesos de producción, distribución y consumo de bienes y servicios, que llamaremos la economía real $^{15}$. Para resolver los problemas asociados con los procesos económicos, el economista cuenta con unas competencias profesionales que ha adquirido en su formación, dentro de las cuales están los elementos que conforman nuestro enfoque de competencias a partir de las disposiciones.

Este conjunto de elementos le permitirá al recién graduado enfrentar los retos o problemas de la economía real y formarse su realidad, es decir, su propio sistema de teorías y modelos mentales privados que debe aprender "a echar a andar" o a "correr (run)" mentalmente con apoyo de sistemas simbólicos y sistemas computacionales para contrastar sus previsiones con la realidad económica, es decir, con otros sistemas públicos de teorías y modelos socialmente compartidos (o al menos dados por compartidos) por los expertos reconocidos entre los practicantes de la disciplina económica y ente los políticos, empresarios y otros dirigentes que guían sus decisiones por las interpretaciones que les dan a esos modelos y teorías.

15 Vasco (2014) define el universo como el agregado de todos los procesos reales que conforman lo real. Cuando hablamos de economía real nos referimos únicamente al agregado de todos los procesos económicos reales, es decir, procesos de producción, distribución y consumo de bienes y servicios. 


\section{En economía, muchas de las} situaciones o problemas que enfrentan los profesionales están relacionados con las decisiones sobre la compra de una nueva máquina dentro de un proceso de producción o con la contratación o despido de trabajadores. En otros casos, se quiere analizar el impacto de un aumento de impuestos sobre los precios de productos básicos de la canasta familiar.

La coordinación apropiada de estos sistemas privados con los públicos le permiten al profesional de la economía contrastar las previsiones, predicciones y conjeturas que formule a partir de los modelos y teorías que active, para intentar resolver un problema $u$ otro reto económico, con la evolución de los procesos de la economía real.

Ese desempeño reflexivo, eficaz y con sentido de las actividades o tareas que le faciliten dar respuesta oportuna a los retos o problemas de la economía real, tanto por los éxitos como por los fracasos, le permitirá ir pasando de novicio a competente y luego a experto, y en algunos casos hasta llegar a un virtuosismo que le abra el camino para llegar a los más difíciles cargos directivos y a los reconocimientos internacionales más honrosos (Acosta y Vasco, 2013).

En economía, muchas de las situaciones o problemas que enfrentan los profesionales están relacionados con las decisiones sobre la compra de una nueva máquina dentro de un proceso de producción o con la contratación o despido de trabajadores. En otros casos, se quiere analizar el impacto de un aumento de impuestos sobre los precios de productos básicos de la canasta familiar. Desde la perspectiva de la toma de decisiones, es necesario saber la conveniencia de una u otra decisión, así como llegar incluso a determinar si las decisiones son óptimas. La gran mayoría de estas situaciones tiene que ver con variaciones de precios y cantidades. Para su análisis, los economistas desarrollan distintos tipos de modelos: modelos analíticos, modelos econométricos, modelos computacionales, modelos gráficos y modelos estadísticos. En la presente investigación estamos interesados en los modelos analíticos, es decir, aquellos que abordan los problemas económicos a través de fórmulas, ecuaciones, funciones, sus propiedades y sus operadores.

En los primeros cursos de fundamentos y en algunos cursos introductorios de microeconomía, algunos profesores al ver que los profesores de cálculo I todavía no han introducido las derivadas parciales y apenas están repasando la noción de límite en las primeras semanas, enseñan primero la marginalidad como diferencia de precios por peso o por dólar, y dejan para después (o nunca explican) la relación con la derivada parcial o total en cálculo.

Sea cual sea el orden de presentación, estamos interesados en los modelos analíticos que abordan los problemas económicos a través de funciones que son diferenciables, ya que en la mayoría de los casos el objeto matemático de la derivada está asociado al concepto económico de marginalidad. En efecto, el concepto de marginalidad ha estado asociado a desarrollos teóricos para abordar múltiples situaciones como: la doctrina del costo de oportunidad, distribución del producto de acuerdo con las productividades marginales de cada uno de los insumos, el concepto de elasticidad como el cociente de las variaciones porcentuales (elasticidad de sustitución entre bienes consumidos o entre insumos), el análisis de la actividad económica (productividad, costos y rendimientos), la caracterización de las soluciones óptimas y subóptimas de problemas de la teoría del equilibrio general. De 
igual forma, la economía aplicada a través de la econometría ha desarrollado métodos para medir la productividad marginal en el análisis de la producción y el crecimiento económico, así como las variaciones porcentuales de un conjunto de variables. Tenemos así que el concepto de marginalidad forma parte del núcleo de la teoría económica ${ }^{16}$.

El concepto de marginalidad asociado a las diferencias o al cociente de diferencias busca medir o cuantificar los cambios que permitan tomar una decisión. Formalmente, el concepto de marginalidad emerge del uso del cálculo en la teoría económica ${ }^{17}$, en particular del concepto de derivada. En efecto, mientras la derivada de una función de una sola variable se define como el límite de un cociente de incrementos, formalmente:

$$
\frac{d y}{d x}=\lim _{\Delta x \rightarrow 0} \frac{f(x+\Delta x)-f(x)}{\Delta x}
$$

El concepto de marginalidad corresponde al cociente discreto del incremento de una unidad adicional en vez de un incremento infinitesimal, que puede ser entendida de dos

16 El concepto de marginalidad en economía fue dado originalmente por Dupuit, Jules (1884), De la mesure de l'utilité des travaux publics, Annales des Ponts et Chaussées, para determinar la tarifa que debería fijarse por la demanda de un puente, es decir su utilización Ekelund (1968, 1969), Ekelund y Hébert (1999).

17 El primer trabajo sobre economía matemática se atribuye al italiano Giovani Ceva en 1711: De re Nummaria, quoad fieri potuit Geometrice Tractata. La Teoría Neoclásica surge a finales del Siglo XIX con la revolución marginalista (Cournot, 1838; Edgeworth, 1881; Jevons, 1871; Pareto, 1906; Walras, 1900). Durante la primera mitad del Siglo XX los trabajos de Hicks (1939) y Von Neumann (1946) contribuyeron a consolidar los fundamentos de la Teoría Neoclásica. Sin embargo, fueron los trabajos de Arrow y Debreu (1954) and Debreu (1959) con su modelo de Equilibrio General y Nash (1950) desde la Teoría de Juegos, los que permitieron la consolidación de la Teoría Económica Moderna. maneras: 1) un módulo de simplificación: de una noción avanzada se pasa simplificada para cuadrar la noción de límite y 2) un módulo de génesis: de una noción discreta elemental se pasa a refinarla para llegar a la noción de límite y derivada.

Formalmente, el concepto de marginalidad podemos expresarlo como:

$$
\frac{f(x+1)-f(x)}{1}
$$

Estamos interesados no solo en el proceso de aprendizaje de la derivada como objeto matemático y cómo a partir de este objeto se desarrolla el proceso de aprendizaje del concepto de marginalidad (o viceversa) dentro de distintos contextos económicos, sino cómo estos elementos permiten el desarrollo de la competencia matemática de modelación analítica en economía.

Dentro del proceso de conformación de la competencia matemática de modelación analítica en economía debemos abordar el proceso gradual del desarrollo de conocimientos, habilidades, actitudes, comprensiones y sensitividad para las detecciones de oportunidades de uso respecto del campo conceptual de la marginalidad.

Es decir, de las magnitudes y de las cantidades relacionadas con la modelación de los procesos económicos que busca integrar el desarrollo de conceptos y problemas en economía a partir del aprendizaje de la derivada como objeto matemático, así como su relación con otros objetos matemáticos como son:

- La variación y su modelación con funciones y su aproximación a través de funciones escalonadas $\mathrm{o}$ funciones $\mathrm{PL}$ (Picewise-Linear).

- Las funciones reales de una o más variables.

- La derivada total y las derivadas parciales.

- La marginalidad y la elasticidad.

- Las magnitudes escalares y vectoriales. 
Gráfico 4. Evaluación de la competencia matemática de modelación analítica en economía

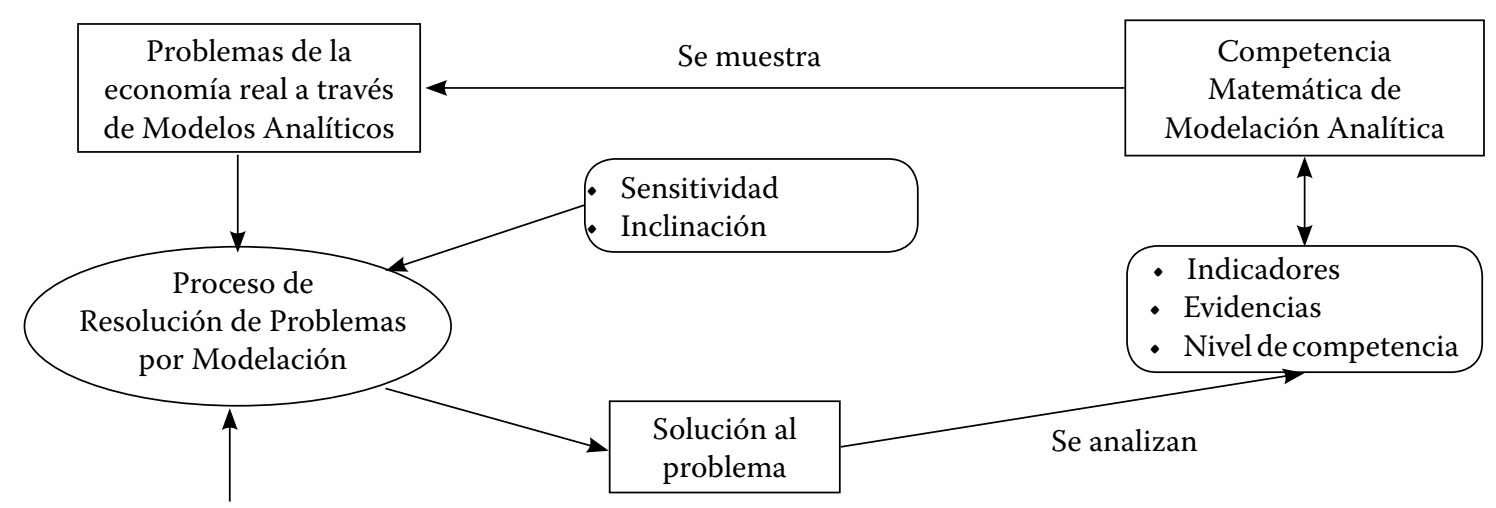

Aptitud

- Modelos y Teorías en Economía

- Configuración de objetos matemáticos asociados a la derivada (Variación y modelación con funciones, funciones reales de una o más variables, derivada total y derivadas parciales, magnitudes escalares y vectoriales, entre otras)

- Conceptos de Economía asociadas al concepto de marginalidad

- Prácticas matemáticas

Capacidad Reflexiva

Fuente: Junca (2018)

Desde la perspectiva del EOS (Enfoque OntoSemiótico de Font et al., 2011) se plantea que las competencias se muestran y desarrollan a través de tareas, mientras que para la EpC (enseñanza para la comprensión) es a través de desempeños. Es así como para evaluarlas es necesario a priori fijar una competencia, diseñar tareas que desarrollen y evidencien dicha competencia, observar si el alumno resolvió esas tareas y, finalmente, analizar la resolución para inferir si el alumno da muestras de la competencia fijada previamente. En la EpC y en el EOS, los mismos tipos de tareas, problemas y situaciones problema son los que se utilizan para desarrollar y evaluar el nivel de desarrollo de las competencias, donde se va construyendo un banco de situaciones y de él a veces se usan unos para aprender y otras para evaluar.

Siguiendo dicho planteamiento, en la Figura 4 se muestra de manera esquemática cómo podría ser la evaluación de la competencia matemática de modelación analítica en economía desde el enfoque de disposiciones de Acosta y Vasco (2013).
Como se muestra en el Gráfico 4, en primer lugar definimos la competencia que queremos evaluar, es decir, la competencia matemática de modelación analítica. Dicha competencia debe mostrarse a través de tareas o situaciones problemáticas en economía que puedan ser abordados por medio de modelos analíticos y que deben presentarse dentro de contextos relativamente nuevos y retadores. Luego los estudiantes demostrarán su grado de competencia en matemáticas en el proceso de resolución del problema propuesto, es decir, mostrarán el conjunto de conocimientos, habilidades, actitudes, comprensiones y disposiciones cognitivas, metacognitivas, socioafectivas y psicomotoras, apropiadamente relacionadas entre sí, para facilitar el desempeño reflexivo, flexible, eficaz y con sentido de una actividad o en este caso de un conjunto de tareas. Allí debemos evidenciar los cuatro pilares de nuestra concepción de competencia: la aptitud, la capacidad reflexiva, la sensitividad y la actitud o inclinación, a través de indicadores y evidencias, para finalmente poder determinar el grado o nivel de competencia del estudiante. 


\section{Conclusiones}

El plantear un modelo de la competencia matemática de modelación analítica en economía implica el desarrollo de dos conjuntos de elementos integradores. En primer lugar, el de la competencia general desarrollada por Acosta y Vasco (2013) y Junca (2018), donde se caracteriza la competencia de manera general a partir de cuatro elementos: la aptitud (qué y cómo), la aptitud, la sensibilidad y la reflexividad. Estos cuatro elementos son presentados en una competencia particular de la modelación matemática del economista.

En este aspecto queremos resaltar cómo la sensibilidad implica no solo el saber el momento justo (timing) para aplicar el qué y el cómo, sino que además implica que ese qué y cómo se muestra de una manera adecuada y rigurosa. Pero más aún, nuestros estudiantes en su formación deben desarrollar la reflexividad de manera que aborden los problemas de la vida real económica (reforma laboral, reforma pensional) y sean críticos de las limitaciones teóricas de los modelos matemáticos que proponen y con los que toman decisiones de política. Más aún, que miren sus implicaciones éticas.

El segundo aspecto que integra esta propuesta de la CMMA en economía es el de la modelación como tal. Es decir, la articulación de un primer momento de representación de la realidad en la teoría económica y la de esta en el mundo de las matemáticas. Para luego trabajar allí y resolver con las matemáticas adecuadas el problema que se quiere resolver, es decir, con herramientas que no son únicamente de optimización como erróneamente nos hemos concentrado por la fuerza de la economía neoclásica dominante. Los problemas complejos implican otro tipo de matemáticas, donde las herramientas del cálculo y la optimización son una alternativa.

Un segundo momento es el de la interpretación de los resultados del modelo matemático en la economía y de la interpretación del modelo económico en los problemas concretos de la realidad. Esta interpretación no puede limitarse a unas conclusiones del impacto del modelo, es decir, del impacto positivo o negativo de un cambio de impuestos o tarifas, sino que implica hacer explícitas las limitaciones de los resultados que parten de las simplificaciones de la realidad en el modelo. $Y$ hacer explícitas las implicaciones éticas de las propuestas de política que se derivan del modelo.

Este desarrollo de la CMMA implica el esfuerzo de formar economistas más integrales en una formación pluralista en economía. Implica diseñar seminarios que permitan enseñar a modelar y no a aplicar modelos como un recetario de cocina.

\section{Referencias}

Acosta, D. \& Vasco, C. (2013). Habilidades, competencias y experticias: Más allá del saber qué y el saber cómo. Bogotá: Corporacion Universitaria Unitec, Universidad de Manizales, Fundación Centro Internacional de Educación y Desarrollo Humano.

Allgood, S. \& Walstad, W. (1999). The Longitudinal Effects of Economic Education on Teachers and Their Students. The Journal of Economic Education, 30(2), 99-111.
Arrow, K. \& Debreu, G. (1954). Existence of an Equilibrium for a Competitive Economy. Econometrica, 22, 265-290.

Asano, A. (2006). Teaching marginal analysis: On the importance of emphasising the second-order condition. International Review of Economics Education, 5(1), 46-59.

Backhouse, R. (1998). If Mathematics Is Informal, Then Perhaps We Should Accept That 
Economics Must Be Informal Too. Economic Journal, 108(451), 1848-1858.

Badillo, E., Azcárate, C., \& Font, V. (2011). Análisis de los niveles de comprensión de los objetos $\mathrm{f}^{\prime}(\mathrm{a})$ y $\mathrm{f}^{\prime}(\mathrm{x})$ en profesores de matemáticas. Enseñanza de las ciencias, 29(2), 191-206.

Ballard, C. \& Johnson, M. (2004). Basic Math Skills and Performance in an Introductory Economics Class. Journal of Economic Education, 35(1), 3-23.

Ballard, C. \& Johnson, M. (2005). Gender, Expectations, and Grades in Introductory Microeconomics at a US University. Feminist Economics, 11(1), 95-122.

Balzer, W. \& Moulines, C. (1996). Structuralist Theory of Science. Berlin: Walter de Gruyter. Balzer, W., Sneed, J., \& Moulines, C. (1987). An Architectonic for Science. The Structuralist Progam. Dordrecht: Reidel Publishing Company.

Beattie, B. R. \& LaFrance, J. T. (2006). The Law of Demand versus Diminishing Marginal Utility. Review of Agricultural Economics, 28(2), 263-271.

Becker, W. (1997). Teaching Economics to Undergraduates. Journal of Economic Literature, 35(3), 1347-1373.

Becker, W., Harter, C., \& Watts, M. (1999). Who Teaches with more than Chalk and Talk? Eastern Economic Journal, 25(3), 343-356.

Benedict, M. \& Hoag, J. (2002). Who's Afraid of Their Economics Classes? Why Are Students Apprehen- sive about Introductory Economics Courses? An Empirical Investigation. American Economist, 46(2), 31-44.

Bennett, J. \& Johnson, M. (1979). Mathematics and Quantification in Economic Literature: Paradigm or Paradox? Journal of Economic Education, 11(1), 40-42.

Bernat, L., Jácome, F., Mejia, R., Torrez, B., \& Torres, E. (2010). Competencias fundamentales en economía. AFADECO. Bogotá: Colombia.

Bustamante, G. (2003). El concepto de Competencia III. Las "competencias" en la educación colombiana. Bogotá: Sociedad Colombiana de Pedagogía - Alejandría Libros.

Bustamante, G., De Zubiria, S., Bacarat, M., Graziano, N., Marín, L., \& Gómez, J. (2004). El concepto de Competencia II. Una mirada interdisciplinar. Bogotá: Sociedad Colombiana de Pedagogía - Alejandría Libros.

Bustamante, G., Torres, E., Marín, L., Gómez, J., \& Barrantes, E. (2002). El concepto de Competencia I. Una mirada interdisciplinar. Bogotá: Sociedad Colombiana de Pedagogía - Alejandría Libros.

Butler, J., Finegan, T., \& Siegfried, J. (1998). Does More Calculus Improve Student Learning in Inter- mediate Micro- and Macroeconomic Theory? Journal of Applied Econometrics, 13(2), 185-202.

Chen, P. (2008). Equilibrium Illusion, Economic Complexity and Evolutionary Foundation in Economic Analysis. Evolutionary Institutional Economic Review, 5(1), 81-127.

Colander, D. \& Klamer, A. (1987). The Making of an Economist. The Journal of Economic Perspectives, 1(2), 95-111.

Colander, D. \& Ñopo, H. (2011). Educating Latin American Economists. International Review of Eco- nomics Education, 10(1), 54-69.

Cournot, A. (1838). Researches into the Mathematical Principles of the Theory of Wealth. New York: A. M. Kelley. 1971.

Debreu, G. (1959). Theory of Value: An Axiomatic Analysis of Economic Equllibrium. New Haven: Yale University Press.

Didia, D. \& Hasnat, B. (1998). The Determinants of Performance in the University Introductory Finance Course. Financial Practice and Education, 8(1), 102-107. 
Diez, J. \& Moulines, C. (1999). Fundamentos de filosofía de la ciencia. Madrid: Ariel. Dubas, J. \& Toledo, S. (2016). Taking higher order thinking seriously: Using marzano's taxonomy in the economics classroom. International Review of Economics Education, 21, 12-20.

Duval, R. (2004). Semiosis y pensamiento humano. Instituto de Educación y Pedagogía. Cali: Universidad del Valle. (Original publicado en Francés en 1995).

Edgeworth, F. (1881). Mathematical Psychics: An Essay on the Application of Mathematics to the Moral Sciences. London: Kegan Paul.

Ekelund, R. (1968). Jules Dupuit and the Early Theory of Marginal Cost Pricing. Journal of Political Economy, 76, 462-471.

Ekelund, R. (1969). A Note on Jules Dupuit and Neo-Classical Monopoly Theory. Southern Economic Journal, 35, 257-262.

Ekelund, R. \& Hebért, R. (1999). The Dupuit-Marshall Theory of Competititive Equilibrium. Economica, 66, 225-240.

Fernández, A., Ed. (2005). Marco de fundamentación conceptual y especificación del ECAES de Economía. Bogotá: AFADECO.

Font, V. (2000). Representaciones ostensivas que pueden ser activadas en el cálculo $\mathrm{f}^{\prime}(\mathrm{x})$. Uno: Revista de didáctica de las matematicas, (25), 21-40.

Font, V. (2005). Representaciones activadas en el cálculo de $\mathrm{f}^{\prime}(\mathrm{x})$. Workin Paper. Barcelona: Universidad de Barcelona. p. 11.

Font, V. (2009). Formas de argumentación en el cálculo de la función derivada de la función $\mathrm{f}(\mathrm{x})=\mathrm{x} 2$ sin usar la definición por límites Vicenç Font Moll. Unión. Revista Iberoamericana de Educación Matemática, (18), 15-28.

Font, V. (2010). Análisis didáctico de objetos y procesos matemáticos. La derivada como contexto de reflexión. Working Paper. Barcelona: Universidad de Barcelona. IX Conferencia Argentina de Educación Matemática. p. 8.

Font, V.\& Ramos, A. (2005). Objetos personales matemáticos y didácticos del profesorado y cambio institucional. El caso de la contextualización de funciones en una facultad de ciencias económicas y sociales. Revista de Educación, (338), 309-345.

Font, V. \& Ramos, A. (2008). Criterios de idoneidad y valoración de cambios en el proceso de instrucción matemática. Revista Latinoamericana de Investigación en Matemática Educativa, 11(2), 233-265.

Font, V., Robles, M., \& Del-Castillo, A. (2011). Análisis y valoración de un proceso de instrucción sobre la derivada. Working Paper. Universidad de Barcelona - Universidad de Sonora p. 28.

Gery, F. (1970). Mathematics and the Understanding of Economic Concepts. Journal of Economic Education, 2(1), 100-104.

Godino, J., Font, V., Pino-Fan, L., \& Castro, W. (2011). Explorando el conocimiento didáctico-matemático de profesores en formación sobre derivada. Working Paper. Barcelona: Universidad de Barcelona. p. 25.

Gorry, D. \& Gilbert, J. (2015). Numerical simulations of competition in quantities. International Review of Economics Education, 18, 49-61.

Grubel, H. \& Boland, L. (1986). On the Efficient Use of Mathematics in Economics: Some Theory, Facts and Results of an Opinion Survey. Kyklos, 39(3), 419-442.

Guest, R. \& Duhs, A. (2002). Economics Teaching in Austalian Universities: Rewards and Outcomes. The Economic Record, 78(241), 147-160.

Hansen, W. (1991). The Education and Training of Economics Doctorates: Major Findings of 
the Executi- ve Secretary of the American Economic Association's Commission on Graduate Education in Economics. Journal of Economic Literature, 29(3), 1054-1087.

Hartman, D. (1978). What do Economics Majors Learn? The American Economic Review, 68(2), 17-22. Hey, J. (2005). I Teach Economics, Not Algebra and Calculus. Journal of Economic Education, 36(3), 292-304.

Heyne, P. (1995). Teaching Introductory Economics. Agenda, 2(2), 149-158.

Hicks, J. (1939). Value and Capital: An Inquiry into some Fundamental Principles of Economic Theory. Oxford: Clarendon Press.

Jevons, W. (1871). The Theory of Political Economy. New York: A. M. Kelley.

Junca, G. (2018). Caracterización de la Competencia Matemática de Modelación Analítica en Económía. El concepto de marginalidad asociado al estudio de la derivada. Doctorado Intersititucional en Educación. Universidad Distrital Francisco José de Caldas.

Kantorovich, L. (1989). Mathematics in Economics: Achievements, Difficulties, Perspectives. American Economic Review, 79(6), 18-22.

Katzner, D. (2003). Why Mathematics in Economics? Journal of Post Keynesian Economics, 25(4), 561-574.

Kherfi, S. (2008). Economic Education in the Middle East: Are the Determinants of Success in Introductory Economics Any Different? Journal of Economic Education, $39,22-40$.

Lagerlof, J. \& Seltzer, A. (2009). The Effects of Remedial Mathematics on the Learning of Economics: Evidence from a Natural Experiment. Journal of Economic Education, 40(2), 115-136.

Lora, E. \& Ñopo, H. (2009). La formación de los economistas en América Latina. Documento de trabajo del BID No. 119.
Ministério-Educação \& INEP (2012). Manual do ENADE 2012. Brasília-DF: Ministério da Educação - INEP.

Ministerio-Educación (1998). Series lineamientos curriculares: Matemáticas. Ministerio de Educación Nacional.

Mockus, A. (1988). Representar y disponer: un estudio de la noción de representación orientado hacia el examen de su papel en la comprensión previa del ser como disponibilidad. Bogota: Universidad Nacional de Colombia.

Mora, J., Ramírez, L., \& Acuña, L., Eds. (2012). La enseñanza de la economía en el contexto colombiano. Bogotá: AFADECO.

Nash, J. (1950). Equilibrium points in N-person games. Proceedings of the National Academy of Sciences, 36(48-49).

Naylor, R. \& Smith, J. (2004). Degree Performance of Economics Students in UK Universities: Absolute and Relative Performance in Prior Qualifications. Scottish Journal of Political Economy, 51(2), 250-265.

Nolan, E. \& Ahmadi-Esfahani, F. (2007). Predicting Performance in Undergraduate Agricultural Econo- mics. Australian Journal of Agricultural and Resource Economics, 51(1), 1-15.

OECD (2012). AHELO Project Update. Working Paper. OECD.

Pareto, V. (1906). Manual de Economía Política: con una introducción a la ciencia social. Mexico: Instituto Politecnico Nacional. 1991.

Perkins, D. \& Richhart, R. (2004). When is good thingking? In D. Dai \& R. Sternberg (Eds.), Motivation, emotion and cognition: Integrative perspectives on intellectual functioning and development chapter When is Good Thingking?, (pp. 351-384). New Jersey: Lawrence Erlbaum Associates. 
Perkins, D., Tishman, S., Richhart, R., Donis, K., \& Andrade, A. (2000). Intelligence in the Wild: A Dispositional View of Intelectual Traits. Educational Psychology Review, 12(3), 269-293.

Pezzino, M. (2016). Understanding strategic competition using numerical simulations and dynamic dia- grams in mathematica. International Review of Economics Education, 22(34-47).

Phillips, P. (2007). Mathematics, Metaphors and Economic Visualisability. Quarterly Journal of Austrian Economics, 10(4), 281-299.

PISA (2009). Assessment Framework. Key competencies in reading, mathematics and science. Programme for International Student Assessment.

Ramos, A. (2006). Objetos personales matemáticos y didácticos del profesorado y cambios institucionales. El caso de la contextualización de las funciones en una facultad de ciencias económicas y sociales. $\mathrm{PhD}$ thesis, Universitat de Barcelona.

Raymond, F., Raymond, A., \& McCrickard, M. (2008). Stuck Behind the Math: Just How Helpful Can One Expect Technology to be in the Economics Classroom? International Review of Economics Education, 7(1), 62-102.

Rigall-I-Torrent, R. (2011). Using ProblemBased Learning for Introducing Producer Theory and Market Structure in Intermediate Microeconomics. International Review of Economics Education, 10(1), 1-15.

Rosser, J. B. J. (2003). Weintraub on the Evolution of Mathematical Economics: A Review Essay. Journal of Post Keynesian Economics, 25(4), 575-589.

Rychen, D. \& Salganik, L. (2001). Defining and Selecting Key Competences. Göttingen: Hogrefe and Huber.

Rychen, D. \& Salganik, L. (2003). Key competencies for a Successful Life and a
Well-Functioning Society. Göttingen: Hogrefe and Huber.

Salemi, M., Siegfried, J., Sosin, K., Walstad, W., \& Watts, M. (2001). Research in Economic Education: Five New Initiatives. American Economic Review, 91(2), 440-445.

Samuelson, P. (1994). The To-Be-Expected Angst Created for Economists by Mathematics. Eastern Economic Journal, 20(3), 267-273.

Siegfried, J., Bartlett, W., Hansen, W., Kelley, A., McCloskey, D., \& Tietenberg, T. (1991). The Status and prospects of the economics major. Journal of Economic Education, 22(3), 197-224.

Siegfried, J. \& Fels, R. (1979). Research on Teaching College Economics: A Survey. Journal of Economic Literature, 17(3), 923-969.

Siegfried, J. \& Stock, W. (2007). The Undergraduate Origins of PhD Economists. Journal of Economic Education, 38, 461-482.

Sproule, R. (2015). An argument for the use of the square-root functional form in teaching undergraduate microeconomics. International Review of Economics Education, 20, 37-45.

Staveley-O'Carroll, J. (2015). The impact of classroom demonstrations and online discussions on student achievement. International Review of Economics Education, 20, 46-58.

Vasco, C. (1995). La teoría general de procesos y sistemas. In E. Aldana, G. García-Márquez, R. Gutiérrez, M. Palacios, \& C. Vasco (Eds.), Misión, Ciencia, Educación y Desarrollo. Educación para el Desarrollo, volume 2 of Informes de Comisionados I. Colección Documentos de la Misión (pp. 377-652). Santafé de Bogotá. Presidencia de la República - Consejería Presidencial para el Desarrollo Institucional - Colciencias.

Vasco, C. (2014). Procesos, sistemas, modelos y teorías en la invistigación educativa. In C. 
Mosquera (Ed.), Perspectivas Educativas. Bogotá: Universidad del Valle - Universidad Distrital Francisco José de Caldas - Universidad Pedagógica Nacional.

Vazquez-Cognet, J. (2008). The production of Mathematical Problems: Diminishing Marginal Returns Experiment. International Review of Economics Education, 7(1), 103-116.

Von Neumann, J. (1946). A Model of General Economic Equilibrium. Review of Economic Studies, 13, 1-9.

Walras, L. (1900). Elementos de economía política pura o Teoría de la riqueza social. Madrid: Alianza Editorial. 1987.
Walstad, W. (2001). Improving Assessment in University Economics. The Journal of Economic Education, 32(3), 281-294.

Walstad, W. \& Allgood, S. (1999). What Do College Seniors Know about Economics? The American Economic Review, 89(2), 350-354.

Walstad, W. \& Allgood, S. (2005). Views of Teaching and Research in Economics and Other Disciplines. The American Economic Review, 95(2), 177-183.

Zweig, M. \& Dawes, W. (2000). Qualitative and Quantitative Methods: A New Design for Introductory Economics. American Economist, 44(2), 30-35. । 